\title{
Improving the Transverse Resolution of Optical Coherence Tomography with a Finite Impulse Response Filter and a Series of Numerically Refocused Images
}

DOI: $10.17691 / \mathrm{stm} 2019.11 .2 .02$

Received June 2, 2018

A.A. Moiseev, PhD, Senior Researcher, Laboratory of Highly Sensitive Optical Measurements:

G.V. Gelikonov, DSc, Head of the Laboratory of Highly Sensitive Optical Measurements;

S.Y. Ksenofontov, PhD, Senior Researcher, Laboratory of Highly Sensitive Optical Measurements;

P.A. Shilyagin, PhD, Senior Researcher, Laboratory of Highly Sensitive Optical Measurements;

D.A. Terpelov, PhD, Junior Researcher, Laboratory of Highly Sensitive Optical Measurements;

I.V. Kasatkina, PhD, Researcher, Laboratory of Highly Sensitive Optical Measurements;

D.A. Karashtin, PhD, Researcher, Department of Geophysical Electrodynamics;

A.A. Sovietsky, Senior Research Assistant, Laboratory of Wave Methods

for Studying Structurally Inhomogeneous Media;

V.M. Gelikonov, DSc, Head of the Department of Nano-Optics and Highly Sensitive Optical Measurements

Federal Research Center Institute of Applied Physics, Russian Academy of Sciences, 46 Ulyanova St.,

Nizhny Novgorod, 603950, Russia

Among the numerous methods for improving the informative value of the optical coherence tomography (OCT), a special place is taken by the methods for increasing the spatial resolution of the resulting images. Increasing the resolution allows one to identify more clinically significant structures in OCT images and thus improve the diagnostic value of OCT. Since the transverse resolution of OCT images is determined by the physical principles different from those for the longitudinal resolution, the ways of their improvement are also different.

The aim of the study is to develop a method for increasing the transverse resolution of OCT by using a finite impulse response (FIR) filter for numerical refocusing and by combining the numerically refocused images.

Results. We have developed a FIR filter able to transfer the focal plane of an OCT image using the data from 17 consecutive measurements of the field scattered by the object. In addition, we propose an original method for automatic synthesis of the final OCT image with an improved transverse resolution over the entire object depth from a series of images having different focal plane positions. This will enable the OCT instrument to produce a sharply focused beam for the scanning and thus obtain images with an improved transverse resolution in the focal plane, restore the required resolution in the out-of-focus areas using numerical transfer of the focal plane, and also synthesize the final OCT image with an improved transverse resolution. The method has been tested with model objects using an OCT device operating at a central wavelength of $1 \mu \mathrm{m}$ with the $60 \mathrm{~nm}$ range and a beam focused onto a spot with a diameter of $5 \mu \mathrm{m}$.

Conclusion. The proposed method of numerically increasing the transverse resolution of OCT images allows one to obtain images with an improved transverse resolution using only a small number of OCT measurements; in the future, it will allow for creating an OCT device that produces high-resolution images in real time.

Key words: optical coherence tomography; OCT; signal processing; OCT image processing; OCT transverse resolution improvement.

\section{Introduction}

Optical coherence tomography (OCT) is a rapidly developing method of non-destructive examination of the internal structure of scattering tissues (including biological). The method allows for resolution up to several micrometers within a depth of $2 \mathrm{~mm}$ into the object $[1,2]$. The standard result of an OCT examination is a cross-sectional image of the object, traditionally called the B-scan, which consists of a series of the scattered field amplitude distributions along the depth, or A-scans.

There are a number of approaches to improving the informative value of OCT results; of those, the most obvious one is to increase the spatial resolution of the device [3-6]. In some applications, high spatial resolution allows the operator to identify more clinically significant structures and thus improve the diagnostic value of the test $[7,8]$. While the longitudinal resolution of OCT is determined by the scanning beam spectral width, the

Corresponding author: Aleksandr A. Moiseev, e-mail: aleksandr.moiseev@gmail.com 
transverse resolution depends on the geometry of this beam. Here, the diffraction properties of the beam create a discrepancy between the requirement of high spatial resolution and the depth of probing.

To date, a number of hardware and software solutions to this discrepancy are known. Using the beams that practically do not change their shape at the depth allows for obtaining images with a resolution equal to the width of the major maximum of such beams [9-12]. However, the presence of lateral maxima and the weak coupling between such beams and the singlemode fiber prevent a wider use of such methods [11]. Recording a series of images with a mechanically transferred position of the focal plane allows one to create an OCT image with an increased transverse resolution by synthesizing the final image from this series of images [13-15]. Such devices though require a sophisticated refocusing system, which complicates their use with flexible endoscopic fibers.

A number of software-based approaches to improving the transverse resolution of OCT have also been proposed. In the technology known as interferometric synthetic aperture microscopy $[16,17]$, the threedimensional Fourier spectrum of the volume OCT data is recalculated into a non-equidistant measurement grid; by performing the inverse Fourier transform, the system creates images with an increased transverse resolution. There are methods of numerical refocusing that compensate for the scanning beam diffraction; there, the so-called phase mask modification is applied to the $2 \mathrm{D}$ spectra of the Fourier OCT images at different depths $[18,19]$ or to the $2 \mathrm{D}$ spectra of the Fourier distributions recorded at different wavelengths [20, 21]. The resulting image is synthesized from a series of images with different positions of the focal plane, which is similar to the hardware-based method for increasing the transverse resolution [21].

The above inventions are applicable only to the OCT data volumes having a sufficient number of measurements at each coordinate. Otherwise, the final images would contain artifacts caused by the edge effects of the discrete Fourier transform, thus making such images inapplicable for diagnostics. Because of that, it may be impossible to visualize images with an increased transverse resolution, since the operator must first collect the necessary data and only then evaluate the processing result, which significantly increases the time of the examination.

In this paper, we discuss the possibility of numerical transferring the OCT image focus within the signal space by using one type of linear digital filters, namely, a finite impulse response (FIR) filter. In the OCT device used in this study (Institute of Applied Physics of the Russian Academy of Sciences, Russia), this filter consisted of only 17 elements; this system then became able to visualize a B-scan with an increased transverse resolution and with some lag behind the recorded data required to record $8 \mathrm{~B}$-scans.

\section{Materials and Methods}

OCT instrumentation. We used an OCT setup with a central wavelength of $1 \mu \mathrm{m}$, a spectral width of $60 \mathrm{~nm}$ and a recording frequency of A-scans of $20,000 \mathrm{~s}^{-1}$. To produce a sharply focused beam, an objective lens with a numerical aperture of 0.1 was used; that provided a transverse resolution of up to $5 \mu \mathrm{m}$ in the air. The device accumulated the OCT data files of A-scans sized at $256 \times 512$; each A-scan contained 256 depth measurements. Recording of one B-scan located along the fast scanning axis and containing 256 measurements, took $13 \mathrm{~ms}$.

FIR filter. It has been shown [20] that the following operation performs the numerical transfer of the focal plane in the OCT data volume:

$$
\begin{aligned}
F_{\Delta z}(x, y, z)=\sum_{k} & \exp (i k z) i F T_{u, v \rightarrow x, y}\left[F T_{x, y \rightarrow u, v}\left[f_{0}(x, y, k)\right] \times\right. \\
& \left.\times \exp \left(-i \Delta z \frac{u^{2}+v^{2}}{4 k}\right)\right]
\end{aligned}
$$

where $F_{\Delta z}$ is the magnitude of the optical field after the numerical transfer of the focal plane to a distance $\Delta z ; x, y$ - the lateral coordinates in the OCT signal space; $z$ is the axial coordinate (depth); $k$ is one of the wave numbers inherent in the OCT unit; $F T_{x, y \rightarrow u, v}$, $i F T_{u, v \rightarrow x, y}$ are the direct and inverse Fourier transforms, respectively; $f_{0}$ is the spectrum measured by the OCT unit; $u, v-$ the transverse coordinates in the Fourier space.

Since the updated OCT devices record the volume data during a period of several tens of seconds, the refocusing is desirable to be based on short data sequences. However, direct application of equation (1) to the OCT data without having the sufficient number of measurements in one of the coordinates, would generate the well-known artifacts, which can be seen from the simulated data presented in Figure 1. In this simulation, it was assumed that $256 \mathrm{~A}$-scans were taken along the fast axis, while the processing along the slow axis was carried out using short sequences equal to 5,9 , or 17 measurements. The Figure also shows the characteristic "tails" that would interfere with each other thereby creating false "structures" that would distort the real image.

To avoid the artifacts, equation (1) can be rewritten in terms of spatial filtering, after which the number of elements of the spatial filter can be reduced. Applying the Fourier transform to the convolution operation, we can rewrite equation (1) as

$$
\begin{gathered}
F_{\Delta z}(x, y, z)=\sum_{k} \exp (i k z) f_{0}(x, y, k) \otimes \\
\otimes i F T_{u, v \rightarrow x, y}\left[\exp \left(-i \Delta z \frac{u^{2}+v^{2}}{4 k}\right)\right] .
\end{gathered}
$$

The convolution kernel width in equation (2) can be limited by the width of the point scatter function of the 


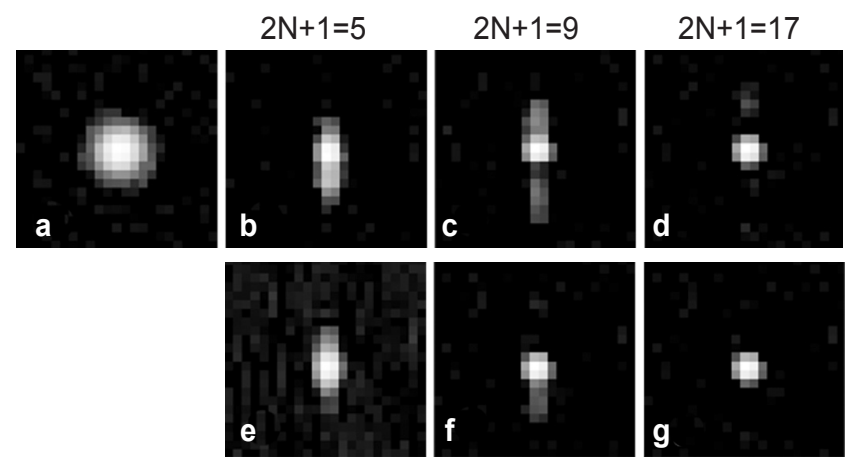

Figure 1. Numerically simulated refocusing based on limited data

Refocusing along the horizontal axis was performed using 256 measurements according to equation (1), while there were 5 , 9, and 17 measurements along the vertical axis (b), (c), (d). Images of the point scatterer were taken at a distance of three Rayleigh lengths from the focal plane with a discretization step of $1 \mu \mathrm{m}$; the size of each image was $30 \times 30 \mu \mathrm{m}$; (a) is the original image; (b)-(d) are images obtained after refocusing along the vertical axis according to equation (1) using 5, 9, and 17 measurements, respectively; (e)-(g) - images obtained after refocusing along the vertical axis according to equation (4) using 5, 9, and 17 measurements, respectively. One can see a decrease in the size of the artifact caused by an insufficient number of measurements if equation (4) is used for each number of measurements; when using 17 measurements the artifact completely disappears

OCT instrument at the respective depth. The convolution kernel is written as follows:

$$
\begin{gathered}
\operatorname{kern}_{\Delta z}\left(x^{2}+y^{2}, k\right)=i F T_{u, v \rightarrow x, y}\left[\exp \left(-i \Delta z \frac{u^{2}+v^{2}}{4 k}\right)\right] \\
\left\{\begin{array}{l}
\sqrt{\frac{2 k}{\Delta z}} \exp \left[i\left(\left(x^{2}+y^{2}\right) \frac{k}{\Delta z}-\frac{\pi}{4}\right)\right], \Delta z>0 \\
\operatorname{kern}_{\Delta z}\left(x^{2}+y^{2}, k\right)=\sqrt{\frac{2 k}{|\Delta z|}} \exp \left[i\left(\left(x^{2}+y^{2}\right) \frac{k}{|\Delta z|}+\frac{\pi}{4}\right)\right], \Delta z<0,
\end{array}\right.
\end{gathered}
$$

where $k e r n_{\Delta z}$ is the kernel of the convolution operation that performs the numerical transfer of the focal plane of OCT images to a distance $\Delta z$.

After their discretization, the values of the kernel (3) at the width of the point scatter function can be considered as the values of an element in the FIR filter. The transfer of the focal plane OCT data can thus be written as

$$
\begin{gathered}
F_{l_{0, j}, n}(\Delta z)=\sum_{m} \exp \left[i\left(k_{0}+\Delta k m\right)\left(z_{0}+d z_{n}\right)\right] \times \\
\quad \times \sum_{l=l_{0}-N}^{l_{0+N}+N} \sum_{j_{0}-N}^{j_{0}+N} f_{l, j, m}(0) k e r n_{l_{0}-l, j_{0}-j, m}(\Delta z),
\end{gathered}
$$

where $I, j, I_{0}, j_{0}$ are the transverse indices of discretization; $n$ is the longitudinal index of discretization; $m$ is the discretization index of the wavenumber; $k_{0}$ is the smallest wavenumber; $\Delta k$ is the wavenumber discretization step; $z_{0}$ is the minimum image depth; $d z-$ the depth discretization step; $F_{l_{0}, j_{0}, n}(\Delta z)$ is the discretized field after the numerical transfer of the focal plane to the distance $\Delta z ; f_{l, j, m}$ is the spectrum measured with the OCT device; $k e r n_{l_{0}-1, l_{0}-j, m}(\Delta z)$ is the discretized convolution kernel that performs the numerical transfer of the focal plane containing $2 \mathrm{~N}+1$ elements.

Equation (4) can be rewritten for the case when the refocusing along one of the coordinates (the fast one) is based on equation (1), and for the second coordinate on equation (4).

Correction for movements. Since the proposed method of numerical refocusing operates with the fullscale data of the scattered field measured with an OCT device, the resulting image is sensitive to phase deviations that could be caused by movements of the object and/or the OCT scanner during the measurement. To compensate for the possible phase deviations, we used the method described in [22].

Synthesis of the final image. Since the specific position of the focal area in the OCT images after refocusing depends on an unknown refractive index of the object under study, it is not known in advance, which of the refocused images (or their parts) are to be selected to create the final image at each depth point. To solve this problem and continue the measurements in the automatic mode, it was proposed to calculate the normalized dispersion that reached its maximum with the optimal focusing at each depth point of each intermediate B-scan [23]:

$$
\text { criterion }\left[F_{l_{0, n}}(\Delta z)\right]=\frac{\operatorname{variance}\left[F_{l_{0}, n}(\Delta z)\right]}{\operatorname{mean}\left[F_{l_{0}, n}(\Delta z)\right]} \text {. }
$$

Since at each depth point, the "criterion" is calculated from a single line, we added a low-frequency filter to reduce the influence of "criterion" fluctuations on the choice of the optimal image. Following this approach, for each depth point of the final image, we selected that part of the intermediate images, which had the maximal "criterion" value at this depth.

\section{Results}

The proposed method of numerical transfer of the focal plane was used to visualize a test biological object: the orange pulp. Figure 2 shows the initial image, the results of refocusing at different depths, as well as the final image, composed of optimally focused intermediate images at the respective depth points. One may notice that the numerical transfer of the focal plane allows for distinguishing the details in out-offocus areas that are inaccessible before refocusing. Only 17 consecutive B-scans were used for refocusing along the slow scanning axis; therefore, if a real-time visualization program based on the described method 


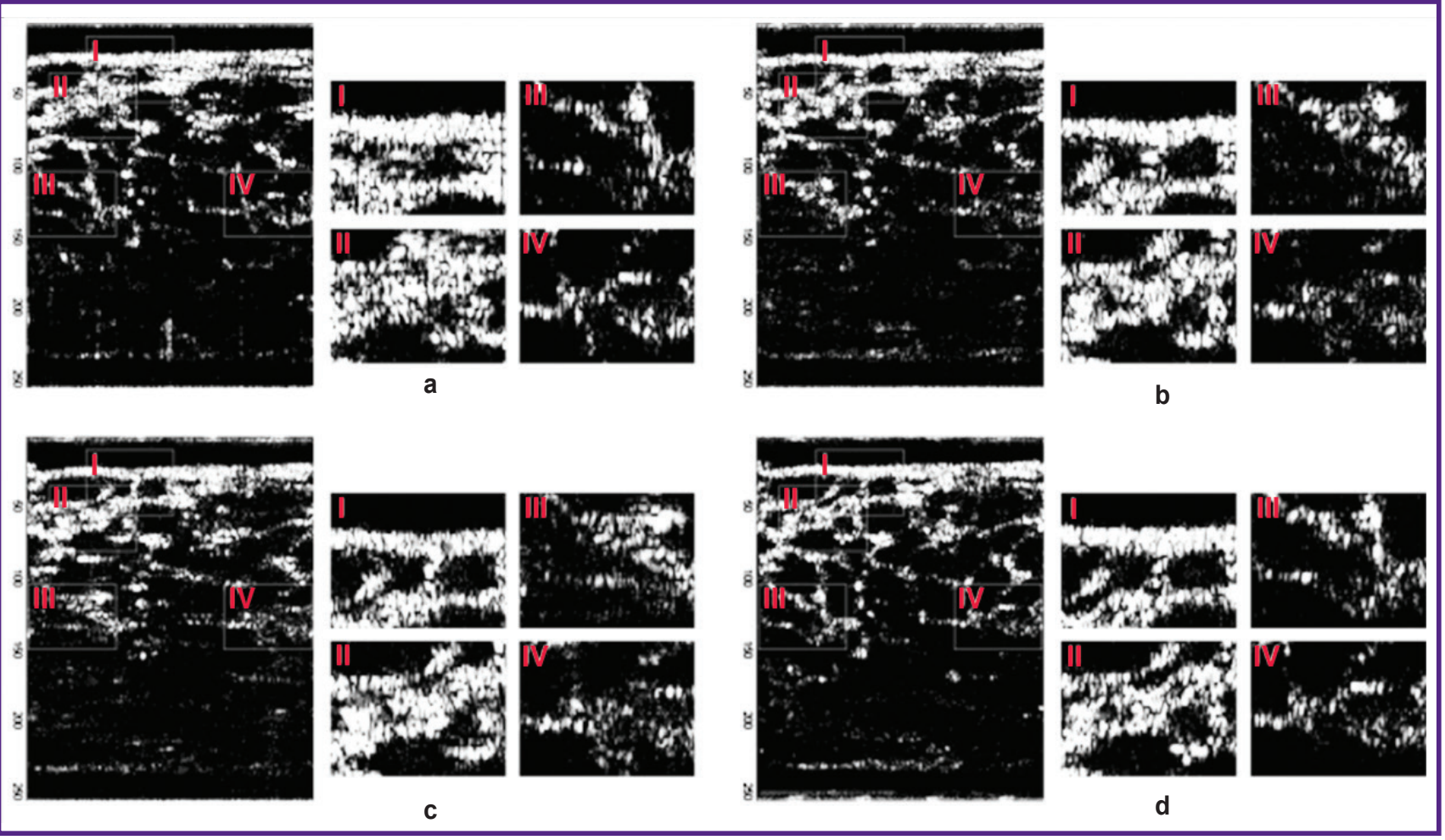

Figure 2. Testing the proposed algorithm with a model object:

(a) original image; (b), (c) images with numerically transferred focus; (d) the final synthesized image after improving its transverse resolution throughout the entire volume under study. The additional panels are sampled near the initial position of the focal plane (III, IV), as well as near the numerically recalculated positions of the focal plane (I, II) in order to demonstrate the changes in the image quality at the respective depth point after the focal plane transfer

is created, the delay between the visualized and recorded B-scans will equal the time needed to record 8 B-scans. For the given OCT device, this time is $100 \mathrm{~ms}$, which is enough to provide the operator with the necessary feedback.

\section{Conclusion}

A method for increasing the transverse resolution of OCT images is proposed; it is based on the numerical transfer of the focal plane of OCT images. The primary image is produced by using a sharply focused beam to scan the object; then, the final image is synthesized from a series of images obtained with various focal planes. In contrast to the existing techniques, the proposed method allows for obtaining final images with an improved transverse resolution by using only $17 \mathrm{~B}$-scans. This advantage can facilitate creation of an OCT system with an improved transverse resolution able to visualize the full cross-sectional image in real time.

Research funding. This work was supported by the Russian Science Foundation, grant No.17-72-20249.

Conflict of interest. The authors confirm the lack of financial or any other conflicting interests that could affect their work.

\section{References}

1. Adhi M., Duker J.S. Optical coherence tomography current and future applications. Curr Opin Ophthalmol 2013; 24(3): 213-221, https://doi.org/10.1097/icu.0b013e32835f8bf8.

2. Vakoc B.J., Fukumura D., Jain R.K., Bouma B.E. Cancer imaging by optical coherence tomography: preclinical progress and clinical potential. Nat Rev Cancer 2012; 12(5): 363-368, https://doi.org/10.1038/nrc3235.

3. Gelikonov V.M., Gelikonov G.V. New approach to crosspolarized optical coherence tomography based on orthogonal arbitrarily polarized modes. Laser Phys Lett 2006; 3(9): 445451, https://doi.org/10.1002/lapl.200610030.

4. Shakhova N.M., Gelikonov V.M., Kamensky V.A., Kuranov R.V., Turchin I.V. Clinical aspects of the endoscopic optical coherence tomography and the ways for improving its diagnostic value. Laser Phys 2002; 12(4): 617-626.

5. Sudheendran N., Syed S.H., Dickinson M.E., Larina I.V., Larin K.V. Speckle variance OCT imaging of the vasculature in live mammalian embryos. Laser Phys Lett 2011; 8(3): 247252, https://doi.org/10.1002/lapl.201010120.

6. Wang S., Larin K.V., Li J., Vantipalli S., Manapuram R.K., Aglyamov S., Emelianov S., Twa M.D. A focused air-pulse system for optical-coherence-tomographybased measurements of tissue elasticity. Laser Phys Lett 2013; 10(7): 075605, https://doi.org/10.1088/1612-2011/10/7/075605.

7. Gambichler T., Schmid-Wendtner M.H., Plura I., Kampilafkos P., Stücker M., Berking C., Maier T. A multicentre 
pilot study investigating high-definition optical coherence tomography in the differentiation of cutaneous melanoma and melanocytic naevi. J Eur Acad Dermatol Venereol 2014; 29(3): 537-541, https://doi.org/10.1111/jdv.12621.

8. Erickson-Bhatt S.J., Nolan R.M., Shemonski N.D., Adie S.G., Putney J., Darga D., McCormick D.T., Cittadine A.J., Zysk A.M., Marjanovic M., Chaney E.J., Monroy G.L., South F.A., Cradock K.A., Liu Z.G., Sundaram M., Ray P.S., Boppart S.A. Real-time imaging of the resection bed using a handheld probe to reduce incidence of microscopic positive margins in cancer surgery. Cancer Res 2015; 75(18): 37063712, https://doi.org/10.1158/0008-5472.can-15-0464.

9. Schulz-Hildebrandt H., Pieper M., Stehmar C., Ahrens M., Idel C., Wollenberg B., König P., Hüttmann G. Novel endoscope with increased depth of field for imaging human nasal tissue by microscopic optical coherence tomography. Biomed Opt Express 2018; 9(2): 636, https://doi.org/10.1364/ boe.9.000636.

10. Verma Y., Divakar Rao K., Mohanty S.K., Gupta P.K. Optical coherence tomography using a tapered single mode fiber tip. Laser Phys Lett 2007; 4(9): 686-689, https://doi. org/10.1002/lapl.200710045.

11. Villiger M., Pache C., Lasser T. Dark-field optical coherence microscopy. Opt Lett 2010; 35(20): 3489, https:// doi.org/10.1364/ol.35.003489.

12. Yin B., Chu K.K., Liang C.-P., Singh K., Reddy R., Tearney G.J. HOCT imaging using depth of focus extension by self-imaging wavefront division in a common-path fiber optic probe. Opt Express 2016; 24(5): 5555, https://doi.org/10.1364/ oe.24.005555.

13. Aguirre A.D., Zhou C., Lee H.-C., Ahsen O.O., Fujimoto J.G. Optical coherence microscopy. In: Optical coherence tomography. Springer International Publishing; 2015; p. 865-911, https://doi.org/10.1007/978-3-319-06419-2_29.

14. Dubois A., Vabre L., Boccara A.-C., Beaurepaire E. High-resolution full-field optical coherence tomography with a Linnik microscope. Appl Opt 2002; 41(4): 805, https://doi. org/10.1364/ao.41.000805.

15. Qi B., Phillip Himmer A., Maggie Gordon L., Victor Yang X.D., David Dickensheets L., Alex Vitkin I. Dynamic focus control in high-speed optical coherence tomography based on a microelectromechanical mirror. Opt
Commun 2004; 232(1-6): 123-128, https://doi.org/10.1016/j. optcom.2004.01.015.

16. Ralston T.S., Adie S.G., Marks D.L., Boppart S.A., Carney P.S. Cross-validation of interferometric synthetic aperture microscopy and optical coherence tomography. Opt Lett 2010; 35(10): 1683, https://doi.org/10.1364/ol.35.001683.

17. Ralston T.S., Marks D.L., Scott Carney P., Boppart S.A. Interferometric synthetic aperture microscopy. Nature Physics 2007; 3(2): 129-134, https://doi.org/10.1038/nphys514.

18. Yasuno Y., Sugisaka J., Sando Y., Nakamura Y., Makita S., Itoh M., Yatagai T. Non-iterative numerical method for laterally superresolving Fourier domain optical coherence tomography. Opt Express 2006; 14(3): 1006, https://doi. org/10.1364/oe.14.001006.

19. Yu L., Rao B., Zhang J., Su J., Wang Q., Guo S., Chen Z. Improved lateral resolution in optical coherence tomography by digital focusing using two-dimensional numerical diffraction method. Opt Express 2007; 15(12): 7634, https://doi.org/10.1364/oe.15.007634.

20. Moiseev A.A., Gelikonov G.V., Terpelov D.A., Shilyagin P.A., Gelikonov V.M. Digital refocusing for transverse resolution improvement in optical coherence tomography. Laser Phys Lett 2012; 9(11): 826-832, https://doi.org/10.7452/ lapl.201210102.

21. Moiseev A.A., Gelikonov G.V., Terpelov D.A., Shilyagin P.A., Gelikonov V.M. Noniterative method of reconstruction optical coherence tomography images with improved lateral resolution in semitransparent media. Laser Phys Lett 2013; 10(12): 125601, https://doi.org/10.1088/16122011/10/12/125601.

22. Moiseev A., Ksenofontov S., Sirotkina M., Kiseleva E., Gorozhantseva M., Shakhova N., Matveev L., Zaitsev V., Matveyev A., Zagaynova E., Gelikonov V., Gladkova N., Vitkin A., Gelikonov G. Optical coherence tomography-based angiography device with real-time angiography B-scans visualization and hand-held probe for everyday clinical use. J Biophotonics 2018; 11(10): e201700292, https://doi. org/10.1002/jbio.201700292.

23. Fonseca E.S.R., Fiadeiro P.T., Pereira M., Pinheiro A. Comparative analysis of autofocus functions in digital in-line phase-shifting holography. Appl Opt 2016; 55(27): 7663, https://doi.org/10.1364/ao.55.007663. 